\title{
Anti-EphA2 Monoclonal Antibody-MMAF Immunoconjugate MEDI-547
}

National Cancer Institute

\section{Source}

National Cancer Institute. Anti-EphA2 Monoclonal Antibody-MMAFImmunoconjugate

MEDI-547. NCI Thesaurus. Code C79836.

An auristatin analogue immunoconjug ate directed against Eph receptor A2 (EphA2)positive cancer cells with potential antineoplastic activity. Anti-EphA2 monoclonal antibody-MMAF immunoconjugate MEDI-547 is generated by conjug ating the fully human IgG1 anti-EphA2 monoclonal antibody (1C1) to the small-molecule microtubule inhibitor monomethyl auristatin phenylalanine (MMAF) via the stable linker maleimidocaproyl (mc) (1C1-mcMMAF). The monoclonal antibody moiety of this agent selectively binds to cells expressing the EphA2 receptor. After internalization and enzymatic cleavage of the immunoconjug ate within the tumor cell cytosol, free MMAF binds to tubulin and inhibits its polymerization, which may result in G2/M phase arrest and tumor cell apoptosis. The cell-surface receptor EphA2, a member of the ephrin family of receptor tyrosine kinases (RT Ks) involved in mammalian development, is overexpressed by a variety of different cancer cell types. 